Introduction

\title{
Où sont les murs? Penser l'enfermement en sciences sociales
}

Mathilde Darley, Camille Lancelevée et Bénédicte Michalon

\section{(2) OpenEdition \\ Journals}

\section{Édition électronique}

URL : http://journals.openedition.org/conflits/18703

DOI : $10.4000 /$ conflits. 18703

ISSN : $1777-5345$

Éditeur :

CCLS - Centre d'études sur les conflits lilberté et sécurité, L'Harmattan

Édition imprimée

Date de publication : 15 octobre 2013

Pagination : 7-20

ISBN : 978-2-343-01909-3

ISSN : $1157-996 \mathrm{X}$

\section{Référence électronique}

Mathilde Darley, Camille Lancelevée et Bénédicte Michalon, « Où sont les murs? Penser l'enfermement en sciences sociales », Cultures \& Conflits [En ligne], 90 | été 2013, mis en ligne le 15 octobre 2013, consulté le 30 mars 2021. URL : http://journals.openedition.org/conflits/18703 ; DOI : https://doi.org/ $10.4000 /$ conflits. 18703 


\section{Où sont les murs? Penser l'enfermement en sciences sociales}

\section{Mathilde DARLEY, Camille LANCELEVÉE, Bénédicte MICHALON}

Matbilde Darley est chargée de recherche CNRS au Centre Marc Bloch de Berlin (UMIFRE 14 - USR 3130). Elle travaille actuellement, dans le cadre du programme ANR TerrFerme (bttp://terrferme.hypotheses.org/), sur les dispositifs d'enfermement des étrangers en Allemagne et s'intéresse particulièrement aux ressorts de l'action juridique associative dans les centres de rétention administrative. Ses recherches portent également sur la prostitution et sa régulation, et notamment sur les politiques de lutte contre la traite des femmes à des fins prostitutionnelles ainsi que sur l'économie du commerce du sexe en zone frontalière.

Contact : matbilde.darley@cmb.bu-berlin.de

Camille Lancelevée est doctorante au laboratoire Iris (UMR 8156-997, Paris) et au Centre Marc Bloch (Berlin). Dans le cadre de sa thèse de sociologie, elle mène des recherches ethnographiques sur la prise en charge psychiatrique et sur les usages sociaux de la psychiatrie en milieu carcéral en France et en Allemagne. Ses recherches ont également porté sur la gestion carcérale de l'intime et sur la mise en place des Unités de Visites Familiales en prison.

Contact : camille.lancelevee@ehess.fr

Bénédicte Michalon est chargée de recherche CNRS à l'UMR ADESS (5185). Ses travaux portent actuellement sur une lecture spatiale de l'enfermement, et plus spécifiquement sur la rétention administrative des étrangers et l'accueil des demandeurs d'asile. Ayant également travaillé sur les questions migratoires, elle s'intéresse particulièrement à l'intersection enfermement/mobilité et à la production d'un ordre interne, qu'elle analyse à partir d'enquêtes de terrain menées en Roumanie. Elle coordonne depuis 2009 le programme TerrFerme (Les dispositifs de l'enfermement. Approche territoriale du contrôle politique et social contemporain), bttp://terrferme.hypotheses.org/ (2009-2014).

Contact:b.michalon@ades.cnrs.fr 
$\mathrm{D}$ epuis quelques années, la France se dote de nouvelles institutions fermées à la frontière du social, du pénal et du médical. Entre 2002 et 2012 a ainsi été programmée la création de plusieurs UHSA 1 censées répondre à l'augmentation drastique du nombre de personnes présentant des troubles mentaux graves en prison 2, l'ouverture d'un Centre Socio-Médico-Judiciaire de Sûreté ${ }^{3}$ (ou rétention de sûreté) à Fresnes, la construction de 200 chambres d'isolement supplémentaires dans les services de psychiatrie ${ }^{4}$ et de cinq nouvelles Unités pour Malades Difficiles ${ }^{5}$, la mise en place de mesures de contention face à la délinquance juvénile (établissements pénitentiaires pour mineurs et centres éducatifs fermés), ou encore la construction de centres de rétention administrative d'envergure inédite, tel celui ouvert au Mesnil-Amelot en août 2011 spécifiquement dédié à l'hébergement des familles en instance d'éloignement du territoire. Dans le même temps, les taux de détention en milieu carcéral n'ont cessé d'augmenter, dépassant le seuil symbolique de 100 personnes détenues pour 100000 habitants ${ }^{6}$. Autant de preuves d'un nouveau « grand renfermement ${ }^{7}$, qui toucherait les sociétés occidentales ? Explorant cette hypothèse, de nombreux travaux récents voient dans les évolutions contemporaines le signe d'un changement de perception de la déviance et des façons de la traiter, et le retour des principes de la première défense sociale ${ }^{8}$ marquée par une volonté de neutralisation des individus présentant un danger pour la société. Ce nouveau « grand renfermement "s’inscrirait plus largement dans le punitive turn repéré aux États-Unis dans le dernier quart du $\mathrm{XX}^{\mathrm{e}}$ siècle. Coïncidant avec le déclin de l'État-Providence 9 , ce tournant punitif serait

1. Unités hospitalières spécialement aménagées, destinées à accueillir des personnes détenues pour des hospitalisations avec ou sans consentement. Prévues par l'article 48 de la Loi d'Orientation et de Programmation de la Justice du 9 septembre 2002, les premières UHSA (Lyon, Toulouse, Nancy, Villejuif) ont ouvert leurs portes entre 2010 et 2012 et au total, 17 UHSA sont programmées, qui devraient proposer 705 places.

2. Falissard B. (ed.), Enquête de prévalence sur les troubles psychiatriques en milien carcéral, Étude pour le Ministère de la Santé (Direction Générale de la Santé) et le Ministère de la Justice (Direction de l'Administration Pénitentiaire), 2004.

3. Loi n²008-174 du 25 février 2008 relative à la rétention de sûreté et à la déclaration d'irresponsabilité pénale pour cause de trouble mental.

4. Circulaire DHOS/O2/F2 n²009-23 du 22 janvier 2009 relative au financement par le Fonds pour la Modernisation des Etablissements de Santé Publics et Privés (FMESPP) du plan d'amélioration de la sécurité des établissements ayant une autorisation en psychiatrie.

5. Circulaire DGOS/PF1/R1 n²010-470 du 28 décembre 2010 qui prévoit l'ouverture de nouvelles Unités pour malades difficiles (UMD). Les nouvelles UMD sont situées à Albi, Monestier Merlines, Sotteville lès Rouen, Châlons en Champagne, Lyon.

6. Tournier P.-V., Arpenter le Champ pénal, Vol. 11, n³25, 3 juin 2013 ; au 1er mai 2013, le taux de détention atteint 103 pour 100000 habitants, et le taux de placement sous écrou 122 pour 100000 habitants. Il y a en France aujourd'hui 67839 personnes détenues pour 57235 places opérationnelles.

7. Foucault M., Histoire de la folie à l'âge classique, Paris, Gallimard, 1972.

8. Marc Ancel, théoricien de la « défense sociale nouvelle " (une défense sociale «impliquant davantage des mesures curatives et rééducatrices que des mesures d'élimination et de neutralisation ») parle de " première défense sociale » pour qualifier les réflexions autour de la réaction sociale au crime développées notamment par Garofalo à la fin du XIXe siècle (Van de Kerchove M., "Risque, dangerosité et sécurité. Renaissance et mutations de la défense sociale ", Déviance et société, vol. $34, n^{\circ} 4,2010$, pp. 479-482).

9. Wacquant L., «Symbiose fatale. Quand ghetto et prison se ressemblent et s'assemblent ", Actes de la Recherche en Sciences Sociales, vol. 3, n¹39, 2001, pp. 31-52. 
caractérisé par une augmentation drastique des taux et des formes d'incarcération 10 et l'instauration de politiques pénales draconiennes ${ }^{11}$ visant avant tout les segments les plus pauvres de la population 12 - lesquels trouveraient paradoxalement dans les institutions de cet «État pénal » les services sociaux et soins démantelés dans le reste de la société ${ }^{13}$. Prolongeant la réflexion, amorcée dès 1939 par Rusche et Kirchheimer ${ }^{14}$ et développée dans les années 1970 par Michel Foucault, sur l'inscription du dispositif d'enfermement et de ses usages dans le contexte socio-politique qui les porte, certains auteurs ${ }^{15}$ ont ainsi posé l'hypothèse que l'enfermement contemporain jouerait un rôle décisif dans la consolidation de l'économie capitaliste. La généralisation de la détention et de l'expulsion comme instruments de contrôle des flux migratoires 16 en fournirait l'exemple le plus criant, témoignant d'un double processus caractéristique de la globalisation économique : le «de-bordering » $\mathrm{du}$ monde occidental, marqué par l'accroissement des circulations humaines, de marchandises et de capitaux notamment, s'accompagnerait d'un mouvement inverse de «re-bordering » des sociétés capitalistes face à certaines mobilités internationales ${ }^{17}$. En conduisant à une criminalisation accrue des migrants ${ }^{18}$, ce processus de réaffirmation des frontières contribuerait à la reproduction d'une force de travail vulnérable, que l'insécurité de son statut rendrait particulièrement adaptable aux besoins de marchés du travail segmentés, typiques des économies post-fordistes.

Ce nouveau « grand renfermement » sonnerait ainsi le glas d'une époque qui, succédant à une ère de fermeture progressive des lieux de confinement

10. «Le taux d'incarcération aux États-Unis qui était de l'ordre de 200 pour 100000 habitants depuis le début du XXe siècle a commencé à augmenter à la fin des années 1980 où il atteint 250, mais la progression s'est maintenue à un niveau très élevé, faisant plus que tripler pour atteindre le niveau de 700 pour 100000 habitants en 2005 » (Lascoumes P., « Ruptures politiques et politiques pénitentiaires, analyse comparative des dynamiques de changement institutionnel », Déviance et Société, vol. 30, n³, 2006, pp. 405-419).

11. Garland D., The Culture of Control: Crime and Social Order in Contemporary Society, Oxford, Oxford University Press, 2001 ; Simon J., Governing through Crime, New York, Oxford University Press, 2007.

12. Wacquant L., Punishing the Poor: The Neoliberal Government of Social Insecurity, Durham, NC, Duke University Press, 2009, p. 6.

13. Comfort M., "Punishment Beyond the Legal Offender", Annual Review of Law and Social Science, vol. 3, n¹, 2007, pp. 271-296 ; Bessin M., Lechien M.-H., Zelem M.-C. et Cambon L., Soigner en prison. Logiques et pratiques des acteurs de la réforme des soins en milieu pénitentiaire. Étude pilote sur deux sites, Rapport de recherche au GIP Mission de recherche «Droit et Justice », GRS, 1997.

14. Kirchheimer O. et Rusche G., Punishment and Social Structure, New York, Russell and Russell, 1939.

15. Voir notamment Christie N., L'industrie de la punition : prison et politique pénale en Occident, Paris, Éditions Autrement, 2003.

16. Bloch A. et Schuster L., "At the Extremes of Exclusion: Deportation, Detention and Dispersal”, Ethnic and Racial Studies, vol. 28, n³, 2005, pp. 491-512.

17. De Giorgi A., "Immigration Control, Post-Fordism, and Less Eligibility. A Materialist Critique of the Criminalization of Immigration across Europe", Punishment E Society, vol. 12, n², 2010, pp. 147-167. Voir aussi Valluy J. (dir.), «L’Europe des camps », Cultures $\mathcal{E}$ Conflits, n57, 2005.

18. De Giorgi A., op. cit. 
depuis l'époque moderne ${ }^{19}$, avait vu de nombreuses transformations des lieux fermés marquées par un relatif décloisonnement et une forme d'ouverture sur l'extérieur. Figure emblématique des murs et portails hermétiquement clos du $\mathrm{XVIII}^{\mathrm{e}}$ et XIXe siècle ${ }^{20}$, l'asile psychiatrique s'était par exemple ouvert, dès la fin du XIXe siècle, non seulement à d'autres disciplines de la science médicale, mais aussi à l'environnement social, et plus encore spatial et urbain dans lequel il tendait de plus en plus souvent à s'inscrire 21. Dans le même temps, le recours à la prison pour punir des comportements délictueux enregistrait une forte baisse, au profit d'autres formes de condamnation (financières notamment ${ }^{22}$ ) s'inscrivant dans un cycle de réformes guidé par la volonté d'apporter une « réponse réductionniste au problème pénitentiaire » 23.

Cette tendance au désenclavement des institutions fermées, amorcée au tournant du $\mathrm{XX}^{\mathrm{e}}$ siècle, est généralement présentée comme intrinsèquement liée à l'intervention croissante d'acteurs extérieurs au sein du dispositif, «forces nouvelles » à même de conduire et/ou contraindre au changement des institutions fermées ${ }^{24}$ caractérisées par leur force d'inertie ${ }^{25}$. Ainsi, en France, c'est sous l'influence d'un « réseau associatif d'action publique » constitué de militants provenant des champs médical, juridique, philanthropique ou encore religieux qu'est adoptée, en 1885, la législation relative à la libération conditionnelle ${ }^{26}$. À partir de la deuxième moitié du Xxe siècle, les interventions d'acteurs extérieurs au sein des lieux d'enfermement se multiplient, tandis que croît le rôle qui leur est dévolu. La mise en place du « service social des prisons » en France en 1945 fait entrer les travailleurs sociaux dans le monde carcéral, tandis que, à la même époque, l'intervention de médecins, psychiatres et psychologues commence à s'institutionnaliser au sein de l'appareil carcéral pour ne cesser, dans les décennies qui suivent, d'y prendre de l'ampleur ${ }^{27}$. À

19. Ammerer G., Bretschneider F. et Weiß A. S. (eds.), Gefängnis und Gesellschaft. Zur(Vor-) Geschichte der strafenden Einsperrung, Leipzig, Leipziger Universitätsverlag, 2003.

20. Voir le texte de B. Eyraud et D. Moreau dans ce numéro. Voir aussi Montlibert (de) C., «La production sociale de l'espace construit : de l'asile au secteur psychiatrique ", Regards sociologiques, n², 1991, pp. 61-80; Coldefy M., De l'asile à la ville : une géographie de la prise en charge de la maladie mentale en France, Thèse de doctorat de géographie, Paris 1, PanthéonSorbonne, 2010, p. 134.

21. Comme le montrent ici les textes de Sophie Ledebur et de Benoit Eyraud et Delphine Moreau.

22. Ammerer G., Bretschneider F. et Weiß A. (eds.), Gefängnis und Gesellschaft. Zur (Vor-) Geschichte der strafenden Einsperrung, op. cit., p. 10.

23. Faugeron C. et Le Boulaire J.-M., " Prisons, peines de prison et ordre public », Revue française de sociologie, vol. 33, n¹, 1992, pp. 3-32. En font également partie les lois sur la relégation, évoquées par Marine Coquet dans ce numéro.

24. Artières P. et Lascoumes P. (eds.), Gouverner, enfermer. La prison, un modèle indépassable?, Paris, Presses de Sciences Po, 2004, reprenant une idée de Simmel G., Sociologie et épistémologie, Paris, PUF, 1981.

25. Lascoumes P., "Ruptures politiques et politiques pénitentiaires, analyse comparative des dynamiques de changement institutionnel ", Déviance et Société, vol. 30, n³, 2006, pp. 405419.

26. Kaluszynski M., "Construire la loi. La Société générale des prisons (1877-1900) », in Kaluszynski M. et Wahnich S. (eds.), L'État contre la politique ? Les expressions historiques de l'étatisation, Paris, L'Harmattan, 1998, pp. 205-221. 
partir des années 1960, les psychiatres jouent également un rôle moteur dans l'élaboration des principes de la sectorisation psychiatrique ${ }^{28}$, devant permettre de dépasser le modèle jusqu'alors prévalent de l'asile psychiatrique pour développer des formes de prise en charge « hors les murs ». La place accordée aux acteurs religieux et aux organisations non gouvernementales et caritatives dans l'accompagnement des personnes enfermées augmente également considérablement, de sorte qu'ils constituent désormais souvent des piliers de l'équilibre du dispositif d'enfermement ${ }^{29}$. Cette dynamique d'ouverture s'accélère à partir des années 1970, avec la popularisation du thème de l'État de droit 30 et la multiplication de mobilisations critiques se concentrant notamment sur les institutions réclusives, pointées pour le manque de transparence qui les caractérise ${ }^{31}$. S'instaure alors la progressive reconnaissance de droits spécifiques aux enfermés, mais surtout la constitution d'une part de groupements militants revendiquant ces droits, et d'autre part de procédures et d'instances destinées à en garantir l'effectivité. Le processus de «détotalisation » des lieux d'enfermement, évoqué par certains auteurs, semble alors passer par l'attribution d'une place plus importante au droit dans les rapports sociaux entre les murs ${ }^{32}$. La montée en puissance des droits des prisonniers et le rapprochement du droit pénitentiaire et du droit commun auraient ainsi permis, dans les années 1990, de faire sortir les établissements carcéraux de leur « état d'exception " ${ }^{33}$. En outre, le contrôle associatif que rend possible l'habilitation des acteurs non gouvernementaux à intervenir régulièrement en milieu fermé ${ }^{34}$, et la création d'organismes, nationaux et européens, chargés de veiller au respect des droits des enfermés dans les lieux de privation de liberté (tels

27. Rostaing C., "A chacun son psy. La diffusion des pratiques psychologiques en prison ", Sociologies Pratiques, vol. 2, n¹7, 2008, pp. 81-94 ; Renneville M., " Psychiatrie et prison : une histoire parallèle ", Annales Médico-Psychologiques, n¹62, 2004, pp. 653-656 ; Milly B., Soigner en prison, Paris, Presses universitaires de France, 2001.

28. Henckes N., Le nouveau monde de la psychiatrie française : les psychiatres, l'État et la réforme des hôpitaux psychiatriques de l'après-guerre aux années 1970, Thèse de doctorat, Paris, École des hautes études en sciences sociales, 2007.

29. Sur le rôle des organisations non gouvernementales dans les centres de rétention français, voir par exemple Fischer N., " Jeux de regards. Surveillance disciplinaire et contrôle associatif dans les centres de rétention administrative », Genèses, vol. 2, n75, 2009, pp. 45-65. Sur l'intervention religieuse en rétention dans le contexte allemand, voir Darley Mathilde, " "Trouvez-vous une femme ici et tout s'arrangera..." L'intervention religieuse auprès d'étrangers placés en rétention ", Genre, sexualité É société, n8, 2012, Adresse : http://gss.revues.org/2523; Darley Mathilde, "Les coulisses de la nation. Genre et ethnicité dans les pratiques d'assistance aux étrangers en situation irrégulière ", Sociétés contemporaines, 2013, à paraître.

30. Salle G., La part d'ombre de l'État de droit : la question carcérale en France et en République fédérale d'Allemagne depuis 1968, Paris, Éditions de l'École des Hautes Études en Sciences Sociales, 2009.

31. Ibid.

32. Rostaing C., «Interroger les changements de la prison. Des processus de déprise et de reprise institutionnelle ", Tracés, vol. 2, n¹7, 2009, pp. 89-108; Bouagga Y., "Rentrer dans le droit commun ? ", Champ pénal/Penal field, vol. VII, 2010, Adresse : http://champpenal.revues.org/7898.

33. Lhuilier D. et Veil C. (eds.), La prison en changement, Ramonville Saint-Agne, Erès, 2000.

34. Notamment délivrée dans les années 1980 aux organisations de défense des droits des étrangers enfermés. Sur l'histoire et les effets de l'intervention de la Cimade dans les centres de rétention français, voir Fischer N., « Jeux de regards... », op. cit. 
que par exemple le Contrôle général des lieux de privation de liberté en France ou le Comité de prévention de la torture du Conseil de l'Europe) seraient autant de formes d'injonction à la transparence permettant de porter « hors les murs » la condition enfermée et agissant ainsi comme un puissant vecteur de transformation potentielle du dispositif 35 .

\section{Où sont les murs ? Désenclaver l'analyse des lieux fermés}

Entre ouverture des établissements à de nouveaux acteurs et création de nouvelles structures d'enfermement, entre humanisation des conditions de vie et sécurisation des lieux fermés, entre protection des droits des usagers et neutralisation des patients / détenus / retenus jugés dangereux, les lieux de privation de liberté résistent à l'application d'une seule grille de lecture par les sciences sociales. Le bref retour esquissé ci-dessus sur les évolutions historiques et contemporaines des institutions fermées suffit en effet à révéler le caractère central de la dialectique ouverture / fermeture dans la manière dont elles ont été pensées, mises en œuvre, aménagées, justifiées et critiquées, quel que soit le contexte politique et historique dans lequel elles s'inscrivent.

Cette dialectique est donc au centre des travaux menés au cours de la dernière décennie, qui cherchent à complexifier la vision, jusqu'alors dominante, d'une prison définie comme un "îlot de réclusion » 36 dans un espace où s'exercerait la liberté d'aller et venir. La dichotomie « dans les murs » / « hors les murs », au cour du projet goffmanien d'appréhension des institutions totales ${ }^{37}$, a progressivement été remise en cause au profit d'une attention nouvelle à l'inscription sociale et spatiale de la prison dans son environnement (notamment urbain), et à leurs influences réciproques ${ }^{38}$. Il s'agissait dès lors d'étudier la prison comme « laboratoire d'analyse du social privilégié » permettant de lire « les principes de structuration des rapports sociaux » 39 , autrement dit de l'appréhender « non pas comme une société mais dans la société » 40 . Ont ainsi été mises en évidence les permanences existant, entre le «dedans » et le «dehors », quant aux ressources et capitaux disponibles et à leurs effets sur les trajectoires sociales, hors et dans l'enfermement. La prison est alors apparue comme la « peine du pauvre » 41 par excellence, la population

35. Les effets de ces injonctions à la transparence sur les institutions de réclusion n'ont pour l'instant été que peu étudiés mais font l'objet d'un projet de recherche de Nicolas Fischer en cours de réalisation.

36. Sparks R., "Can Prisons be Legitimate? Penal Politics, Privatization and the Timeliness of an Old Idea”, British Journal of Criminology, vol. 34, n5, 1994, pp. 14-28.

37. Goffman plaçait la frontière dedans/dehors au coeur de sa définition des asiles comme « institution totale ». Goffman E., Asiles : études sur la condition sociale des malades mentaux et autres reclus, Paris, Éditions de Minuit, 1968.

38. Combessie Ph., Prisons des villes et des campagnes : étude d'écologie sociale, Paris, Éditions de l'Atelier, 1996 ; Salle G., « De la prison dans la ville à la prison-ville. Métamorphoses et contradictions d'une assimilation ", Politix, ${ }^{\circ} 97,2012$, pp. 75-98.

39. Faugeron C., Chauvenet A. et Combessie P., Approches de la prison, Bruxelles, De Boeck, 1996.

40. Combessie P., Sociologie de la prison, Paris, La Découverte, 2001. 
carcérale se distinguant souvent par l'absence combinée de différents capitaux (sociaux, professionnels, économiques, culturels, relationnels, etc.) influençant de manière décisive les trajectoires sociales des personnes détenues mais également la vie quotidienne en situation d'enfermement. Le constat de ces continuités entre l'intérieur et l'extérieur des murs, théorisées par Loïc Wacquant sous le terme de « continuum carcéral » 42, a conduit à « décarcéraliser » 43 l'approche de l'enfermement par les sciences sociales. Il s'agissait ainsi non plus d'appréhender la peine de prison comme un «temps à part du système de vie " ${ }^{44}$, mais au contraire de la resituer dans un cadre d'expérience élargi aux trajectoires sociales des enfermés ${ }^{45}$, ainsi que dans le dispositif de «contrôle social institutionnalisé » 46 plus vaste dans lequel elle s'inscrit. De même, tandis que certains travaux s'attachaient à questionner les effets de l'enfermement sur les pratiques et représentations quotidiennes de migrants en situation irrégulière ayant fait l'expérience de la rétention ${ }^{47}$, la désinstitutionnalisation psychiatrique amenait notamment à s'interroger sur les parcours de patients hors des murs de l'hôpital ${ }^{48}$.

Les textes réunis dans ce numéro s'inscrivent directement dans ce projet scientifique de décentrement du regard porté sur les lieux fermés, en étudiant la manière dont contexte et lieu d'enfermement sont mutuellement travaillés l'un par l'autre. L'analyse livrée par Fanny Le Bonhomme, traitant de l'influence du parti communiste sur le quotidien des soignés et des soignants dans un hôpital psychiatrique de Berlin-Est dans les années 1960, permet ainsi de poursuivre les pistes de réflexion esquissées ailleurs sur les liens entre enfermement et politique ${ }^{49}$. L'auteure s'intéresse à l'articulation entre l'institution psychiatrique et la société environnante dans le contexte dictatorial de la

41. Marchetti A.-M., Pauvretés en prison, Ramonville, Editions Érès, 1997. Voir aussi Aubusson de Cavarlay B., «Hommes, peines et infractions : la légalité de l'inégalité », L'Année sociologique, $\mathrm{n}^{\circ} 35,1985$, pp. 275-309.

42. Loïc Wacquant recourt notamment à ce terme à propos de la sur-représentation des Noirs dans les prisons américaines et postule que le système carcéral endosserait désormais les fonctions d'instrument de contrôle de "caste » et de contention des Afro-Américains qu'exerçait jusqu'alors le ghetto. Voir Wacquant L., «Symbiose fatale... », op. cit.

43. Chantraine G., Par-delà les murs: expériences et trajectoires en maison d'arrêt, Paris, PUF Le Monde, 2004.

44. Bouhnik P. et Touzé S., Hérö̈ne, sida, prison: trajectoires, systèmes de vie et rapport aux risques des usagers d'héroine incarcérés, Paris, RESSCOM, 1996.

45. Fernandez F., Emprises. Drogues, errance, prison: figures d'une dépendance totale, Bruxelles, Larcier, 2010; Chantraine G., Par-delà les murs : expériences et trajectoires en maison d'arrêt, Paris, PUF - Le Monde, 2004 ; Cardi C., «Le contrôle social réservé aux femmes : entre prison, justice et travail social », Déviance et Société, vol. 31, n¹, 2007, pp. 3-23.

46. Cardi C., La déviance des femmes. Délinquantes et mauvaises mères : entre prison, justice et travail social, Thèse de doctorat, Université Paris Diderot - Paris 7, 2008.

47. Le Courant S., «Ce que fait la politique de contrôle de l'immigration ", Champ pénal/Penal field, 2010, vol. VII. URL : http://champpenal.revues.org/7889 [Consulté le : 20 juin 2013].

48. Velpry L., Le quotidien de la psychiatrie : sociologie de la maladie mentale, Paris, Armand Colin, 2008.

49. Jobard, F., «L'ajustement et le hiatus. La prison allemande au cours de l'unification » in Artières P. et Lascoumes P. (eds.), Gouverner, Enfermer. La prison, un modèle indépassable?, Paris, Presses de Sciences Po, 2004, pp. 83-110. 
RDA. À partir d'un important travail d'archives au sein de la clinique psychiatrique de la Charité (Berlin-Est), elle analyse la manière dont les catégories d'ordonnancement ayant cours hors les murs, et relatives notamment à l'appartenance ou non au parti communiste, modèlent la relation soignants / patients dans les murs de la clinique. Revenant sur les archives du même hôpital au siècle précédent, le texte de Sophie Ledebur examine comment la première clinique neuropsychiatrique, créée en 1871 à Berlin, a permis de redéfinir les liens entre disciplines médicales et, partant, la forme des soins dispensés, mais aussi de faire émerger des symptômes et des maladies jusqu'alors ignorés et que l'ouverture de la clinique sur son environnement urbain a contribué à révéler. Autrement dit, resituer l'institution dans le contexte médical et surtout urbain qui l'a vue naître et questionner ces effets contextuels sur le comportement des soignants comme des patients permet ici d'éclairer la manière dont l'ouverture de l'institution psychiatrique à d'autres publics a conditionné à la fois l'émergence d'une discipline, la neuropsychiatrie, et la catégorisation des pathologies qui lui sont associées. A la même époque (fin du XIXe siècle), Marine Coquet questionne la construction conjointe du bagne et de la ville de Saint-Laurent-du-Maroni, en Guyane française, et les intrications que ce double projet urbain / carcéral singulier fait naitre entre les structures sociales dans et hors les murs. Elle interroge alors, à la lumière de cette configuration singulière, la pertinence de la notion goffmanienne d'institution totale pour penser la carcéralisation coloniale. Dans une perspective contemporaine, Caroline Touraut revient sur la manière dont l'ouverture des prisons aux proches de détenus a certes contribué à conférer une certaine porosité à la frontière entre intérieur et extérieur de l'institution, mais aussi à étendre l'emprise et le stigmate carcéraux au-delà des murs. La comparaison proposée par David Scheer, dans le contexte belge, entre un établissement pénitentiaire édifié en 1876 et un établissement récent de 2002, questionne la capacité de ce dernier à répondre aux critiques formulées à l'encontre du caractère défensif et déshumanisant des dispositifs de fermeture et de contrôle des établissements plus anciens. L'ethnographie des pratiques des agents de contrôle et des personnes détenues révèle en effet des formes d'appropriation du lieu transcendant largement les politiques initiales d'ouverture ou de fermeture ayant accompagné leur édification et/ou leur aménagement. Dans une perspective historique plus longue, Benoît Eyraud et Delphine Moreau se penchent sur le développement successif et concomitant de politiques d'ouverture et de fermeture de l'institution psychiatrique, évolutions morphologiques qui s'articulent à des transformations du droit et des pratiques de soin. S'appuyant sur l'analyse des archives de l'hôpital du Vinatier, les auteurs questionnent les incidences, sur l'enfermement psychiatrique et ses usages, du dispositif spatial, légal et discursif censé l'encadrer. La restitution des évolutions historiques de ce dispositif depuis le XIXe siècle et l'étude de sa mise en pratiques permettent d'observer la manière dont les acteurs médicaux composent avec les politiques successives d'ouverture et de fermeture du lieu et justifient leurs arrangements avec le droit. 
Autrement dit, se saisissant du paradoxe (souvent souligné par les travaux de sociologie carcérale) relatif au caractère « définitivement archaïque et résolument moderne » 50 des institutions d'enfermement, les textes proposés ici interrogent les effets des évolutions dites «modernisatrices » du dispositif spatial et/ou législatif régissant l'enfermement sur les pratiques au sein des lieux concernés, et questionnent par là même la pertinence de la dialectique ouverture / fermeture pour penser les pratiques quotidiennes du lieu fermé.

\section{Enfermement et (im)mobilités : contrôler les circulations, gouverner les populations?}

Le projet de dépassement de la dialectique ouverture / fermeture et de son corollaire immobilisation / mobilités passe également par une réflexion sur la spatialité de l'enfermement, lue au prisme des mobilités ${ }^{51}$. Le concept de «mobilité gouvernementale ${ }^{52}$, notamment, a permis de saisir la tension entre mobilité et enfermement à l'œuvre dans les institutions de privation de liberté : il englobe en effet tous les types de déplacements des populations enfermées émaillant la période de réclusion, et qui sont décidés et organisés par l'autorité. Qu'elles soient liées à la routine de l'institution (procédures, répartition des enfermés entre les établissements, etc.) ou punitives ${ }^{53}$, ces mobilités gouvernementales perturbent et/ou transforment en effet les institutions et les rapports sociaux en leur sein ${ }^{54}$. En ce sens, la mobilité est un élément constitutif du dispositif de confinement, au même titre que l'immobilisation : le caractère contraignant et/ou punitif de l'enfermement ne réside pas nécessairement dans l'impossibilité de la mobilité, mais peut à l'inverse se nicher dans le déplacement et le contrôle de celle-ci-ce dont atteste l'usage de la surveillance électronique ${ }^{55}$. La mobilité des personnes recluses peut même

50. Chantraine G., Par-delà les murs..., op. cit., p. 1.

51. Gilmore R. W., Golden Gulag: Prisons, Surplus, Crisis, And Opposition in Globalizing California, University of California Press, 2007.

52. Gill N., "Governmental Mobility: The Power Effects of the Movement of Detained Asylum Seekers around Britain’s Detention Estate”, Political Geography, n²8, 2009, p. 187.

53. Michalon B., «La mobilité au service de l'enfermement ? Les centres de rétention pour étrangers en Roumanie ", Géographie et cultures, n81, 2012, pp. 89-108 ; Michalon B., "Mobility and Power in Detention. The Management of Internal Movement and Governmental Mobility in Romania”, in Moran D., Gill N. et Colon D. (eds.), Carceral Geographies: Mobility and Agency in Imprisonment and Migrant Detention, Ashgate, Farnham, 2013, pp. 37-55. Sur les transferts organisés par l'administration pénitentiaire pour «briser toute tentative de reconstruction de liens et d'ébauche de plan pouvant aboutir à une nouvelle tentative [d'évasion] », voir Chantraine G., Par-delà les murs..., op. cit., p. 125.

54. Gill N., "Governmental Mobility: The Power Effects of the Movement of Detained Asylum Seekers around Britain’s Detention Estate”, Political Geography, n²8, 2009, pp. 186-196; Gill N., "Longing for Stillness: the Forced Movement of Asylum Seekers", M/C Journal: A Journal of Media and Culture, 2009, vol.12, $\mathrm{n}^{\circ} 1$. URL : http://www.journal.mediaculture.org.au/index.php/mcjournal/article/viewArticle/123 [Consulté le 20 juin 2013]. Voir aussi Cliquennois G., "Vers une gestion des risques légitimante dans les prisons françaises ?", Déviance et Société, vol. 30, n³, 2006, pp. 355-371; Moran D., Piacentini L. et Pallot J., "Disciplined Mobility and Carceral Geography: Prisoner Transport in Russia”, Transactions of the Institute of British Geographers, vol. 37, n³, 2012, pp. 446-460.

55. Gill N., "Mobility Versus Liberty? The Punitive Uses of Movement Within and Outside 
devenir l'objectif premier d'institutions saturées telles que les urgences psychiatriques qui, confrontées à un dispositif psychiatrique lacunaire, consacrent une large partie de leur activité quotidienne à « produire des lits vides » (produce empty beds ${ }^{56}$ ).

Cette approche du confinement comme un dispositif mouvant, aux frontières floues ${ }^{57}$, et pouvant se localiser dans les espaces de la mobilité ${ }^{58}$, invite également à penser les effets de seuil internes au lieu d'enfermement. L'ordre interne des institutions fermées s'organise en effet, en grande partie, autour de la gestion des flux de personnes ${ }^{59}:$ si la configuration matérielle des lieux est généralement pensée pour contrôler, canaliser, freiner voire empêcher, par des formes de discontinuités architecturales ${ }^{60}$, ces déplacements, il s'agit aussi parfois de les fluidifier et de les encourager. Relevant aussi du libre arbitre de chacun, ces mouvements illustrent également les marges de manœuvre qui existent en ces lieux, dans les limites définies et/ou tolérées par l'institution. Le texte de Sophie Ledebur, dans ce numéro, sur la polyclinique de la Charité à Berlin à la fin du XIXe siècle, révèle comment cette institution nouvelle dans le paysage médical a permis d'organiser la mobilité des patients, en réponse tant à une volonté d'ouverture de l'institution sur son environnement qu'aux tentatives de la discipline psychiatrique d'atteindre une patientèle nouvelle (et, par là même, de potentiels signes cliniques nouveaux) que les asiles fermés n'avaient pas permis d'appréhender. Le texte de Benoît Eyraud et Delphine Moreau traitant des évolutions, sur près d'un siècle, de l'usage de la contrainte au sein de l'asile de Bron révèle lui aussi l'organisation institutionnelle de cir-

Carceral Environments", in Moran D., Gill N. et Colon D. (eds.), Carceral Geographies: Mobility and Agency in Imprisonment and Migrant Detention, Ashgate, Farnham, 2013, pp. 19-35.

56. Rhodes L., Emptying Beds: The Work of an Emergency Psychiatric Unit, Berkeley, University of California Press, 1995, p. 14.

57. Allspach A., "Landscapes of (Neo-)Liberal Control: the Transcarceral Spaces of Federally Sentenced Women in Canada", Gender, Place E Culture, vol. 17, n6, 2010, pp. 705-723; Baer L. D. et Ravenberg B., "The Outside and Inside in Norwegian and English Prisons", Geografiska Annaler, vol. 90, n², 2008, pp. 205-216 ; Comfort M., "In The Tube At San Quentin. The 'Secondary Prisonization' of Women Visiting Inmates", Journal of Contemporary Ethnography, vol. 32, n¹, 2003, pp. 77-107 ; Comfort M., Doing Time Together: Love and Family in the Shadow of the Prison, Chicago, University of Chicago Press, 2008 ; Milhaud O., Séparer et punir. Les prisons françaises : mise à distance et punition par l'espace, Thèse de doctorat, Bordeaux, Université Michel de Montaigne-Bordeaux III, 2009. Voir aussi l'analyse des déplacements contraints comme mode de contrôle, par les employeurs, de la main d'œuvre étrangère (Bruslé T., "Être migrant, rester paysan ? Constructions territoriales et appartenances spatiales des migrants temporaires népalais en Inde », in Dupont V. et Landy F. (eds), Circulation et territoire dans le monde indien contemporain, Paris, Purushartha, EHESS, 2010, pp. 161-188 ; Zeneidi D., « Migrations circulaires et déni de reconnaissance, l'expérience de l'injustice spatiale des saisonnières agricoles marocaines à Huelva (Espagne) ", Justice spatiale, Spatial Justice, n³, 2011, pp. 1-22).

58. Voir Kobelinsky C., Makaremi C. (eds), «Confinement des étrangers : entre circulation et enfermement ", Cultures $\mathcal{E}$ Conflits, $\mathrm{n}^{\circ} 71,2008$.

59. Kantrowitz l'a démontré dès les années 1960-1970, voir Kantrowitz N., Close Control. Managing a Maximum Security Prison: the Story of Ragen's Stateville Penitentiary, New York, Harrow and Heston, 1996.

60. Milhaud O., Séparer et punir..., op. cit. 
culations au sein de l'établissement, dont l'ampleur varie notamment en fonction de la participation des patients à l'activité économique de l'établissement. Enfin, sur la période contemporaine, le texte de David Scheer traite également des circulations et de leurs usages institutionnels dans les prisons contemporaines belges : il y analyse notamment les stratégies déployées par les acteurs du contrôle pour éviter le stationnement des détenus en certains points stratégiques du lieu fermé, tout en mettant en lumière les mobilités différenciées, au sein du lieu d'enfermement, en fonction du statut des enfermés.

\section{Ouvrir et (re)fermer les lieux fermés : de l'apparent paradoxe au conti- nuum stratégique?}

Entre immobilisation et mobilités, ouverture et fermeture, humanisation et sécurisation, « déprise » et " reprise » institutionnelle ${ }^{61}$, ce que traduisent finalement les développements présentés ci-dessus, c'est non seulement la complexité des politiques de l'enfermement comme mode de gouvernement des populations mais aussi le défi que leurs transformations progressistes et néolibérales récentes posent aux sciences sociales. Si l'on garde l'exemple français précédemment évoqué, la dépénalisation de certaines infractions (l'adultère en 1975, l'homosexualité en 1982), le développement d'alternatives à l'enfermement carcéral 62 ou l'abolition de la peine de mort s'inscrivent en effet dans le même mouvement que l'introduction de nouvelles peines incompressibles 63 et participent donc d'un processus ambivalent de "décarcéralisation » tout autant que de renforcement/d'extension de la contrainte carcérale. Exemple emblématique, le bracelet électronique, présenté par l'administration pénitentiaire française comme une alternative à la détention en établissement carcéral et destiné à surveiller les déplacements des condamnés hors détention, aurait pour corollaire une transformation profonde de la dimension spatiale de l'enfermement ${ }^{64}$. Loin de remplacer l'espace carcéral classique, la surveillance électronique s'y ajouterait, "relan(çant) et étend(ant) la gestion carcérale de l'espace » 65. Au-delà de l'écartèlement apparent entre humanisation et sécurisation, on serait donc en présence d'une forme de gouvernementalité inédite et intrinsèquement cohérente, qualifiée par certains de « post-disciplinaire » 66. Concomitante d'un mouvement de transparence accrue des lieux fermés et de multiplication des acteurs impliqués, cette nouvelle forme de

61. Rostaing C., «Interroger les changements de la prison. Des processus de déprise et de reprise institutionnelle », Tracés, vol. 2, n¹7, 2009, pp. 89-108.

62. Voir par exemple l'introduction dans le droit français du travail d'intérêt général en 1983 et du placement sous surveillance électronique en 1997.

63. Snacken S. et Tubex H., « L'évolution des longues peines... Aperçu international et analyse des causes ", Déviance et société, vol. 19, n², 1995, pp. 103-126.

64. Razac O., "Le bracelet électronique et la virtualisation de l'enfermement », Cultures et Sociétés, $\mathrm{n}^{\circ} 10,2009$, pp. 52-57.

65. Razac O., «Le placement sous surveillance électronique mobile (PSEM) : un nouvel espace de la peine ?", Cabiers sur la sécurité, n¹2, 2010, p.209.

66. Chantraine G., «La prison post-disciplinaire », Déviance et Société, vol. 30, n³, 2006, pp. 273-288. 
gouvernementalité resterait toutefois marquée par la mainmise de l'administration des lieux fermés. Autrement dit, l'ouverture des lieux de confinement à de nouveaux acteurs aurait certes conduit l'administration à leur concéder " une prise sur l'exercice de sa force », mais en s'assurant cependant " la mầtrise de leur intervention » 67 . Surtout, cet enfermement " post-disciplinaire » s'articulerait désormais autour d'une gestion actuarielle des déviances visant à écarter les groupes à risque ${ }^{68}$. A une " gestion différenciée des illégalismes » 69 arrimée aux caractéristiques sociales des groupes concernés auraient ainsi succédé des formes différenciées de régulation des déviances reposant cette fois sur une " gestion prévisionnelle des populations » 70 et des risques dont elles sont potentiellement porteuses.

Entre les murs, l'administration pénitentiaire organise également un tri de la population pénale qu'elle peut désormais affecter dans des régimes de détention différenciés 71 pouvant à la fois signifier un allègement de la contrainte et/ou une amélioration du confort pour certains groupes de détenus et, au contraire, une sécurisation accrue des conditions de détention pour d'autres. Le modèle du «Supermax » (ou «control units », développées dans les prisons fédérales américaines depuis les années 1980) ${ }^{72}$, au sein duquel les personnes détenues sont soumis à des formes extrêmes de déprivation sensorielle et de surveillance, constitue une forme archétypale de ces spécialisations spatiales. Loin de conclure à une équation entre accès aux droits, ouverture et humanisation des lieux, on peut donc au contraire poser l'hypothèse que l'augmentation de la place accordée aux procédures légales a pu / peut également servir à justifier un recours accru à la contrainte - en témoigne notamment l'analyse livrée dans ce numéro par Benoît Eyraud et Delphine Moreau à propos de l'hôpital psychiatrique.

Différents travaux en sociologie de la prison ont d'ailleurs montré que l'augmentation du niveau de confort et de la place faite aux droits des détenus dans les nouvelles prisons pouvait venir pallier l'absence de perspectives réha-

67. Ibid.

68. Feeley M. et Simon J., “The New Penology: Notes on the Emerging Strategy of Corrections and its Implications”, Criminology, vol. 30, n4, 1992, pp. 449-474.; Mary Ph., "Pénalité et gestion des risques : vers une justice “actuarielle” en Europe », Déviance et Société, vol. 25, $\mathrm{n}^{\circ} 1,2001$, pp. 33-51.

69. Sur le sens conféré par Foucault à cette notion, voir Foucault M., Surveiller et Punir. Naissance de la prison, Paris, Gallimard, 1975. Sur la pertinence de la notion de " gestion différenciée des illégalismes » pour penser les rapports de domination contemporains dans lesquels sont pris l'ensemble des individus aux prises avec l'Etat, voir Fischer N., Spire A. (eds.), «L’Etat face aux illégalismes », Politix, vol. 22, n³, 2009, pp. 7-20.

70. Castel R., La gestion des risques : de l'anti-psychiatrie à l'après-psychanalyse, Paris, Éditions de Minuit, 1981.

71. Cliquennois G., "Vers une gestion des risques légitimante dans les prisons françaises? ", Déviance et Société, vol. 30, n³, 2006, pp. 355-371.

72. Rhodes L., Total Confinement: Madness and Reason in the Maximum Security Prison, Berkeley, University of California Press, 2004 ; Bouagga Y., "Qui sont les “pires des pires” ? Des usages des classifications en Supermax aux États-Unis », Déviance et Société, vol. 34, n², 2010, pp. 201-216. 
bilitatrices ${ }^{73}$, voire conforter une "définition atomiste " 74 des personnes détenues, pourvues de droits plutôt que membres d'une collectivité. L' «individualisation » des stratégies et l'instrumentalisation intensifiée des rapports sociaux en prison qui en découleraient ${ }^{75}$ s'avèreraient alors incompatibles avec l'avènement de la "paix sociale » et favoriseraient même, au contraire, l'irruption de la violence en prison ${ }^{76}$. Le texte de David Scheer, s'appuyant sur une ethnographie comparée de deux prisons belges, montre ainsi que les prisons modernes, promesses d'un confort accru tant pour les détenus que pour les agents pénitentiaires, sont également porteuses de nouvelles conflictualités. Dans le même ordre d'idées, la construction contemporaine d'un espace intime dans le dispositif carcéral a certes été présentée comme une forme d'humanisation de la sanction, mais différents travaux ont montré que cet aménagement avait également pour effet un contrôle accru, et des formes d'injonction biographique pour les personnes incarcérées 77. L'ouverture (relative) de l'institution pénitentiaire aux proches de détenus (au nom du droit à la vie privée) a en outre également eu pour effet d'augmenter la contrainte pesant sur ces derniers ${ }^{78}$ (en termes de responsabilité et de contrôle sur la trajectoire et l'éventuelle réinsertion du détenu notamment) - sans par ailleurs les dégager de l'emprise du "stigmate carcéral » ${ }^{79}$, comme le montre l’analyse développée par Caroline Touraut dans ce numéro.

Réunir ici historiens, sociologues et criminologues procède donc de la volonté, initiée lors d'un colloque organisé à Berlin en décembre 2011 80, d'instaurer un dialogue encore balbutiant entre les disciplines pour penser

73. Chantraine, G., « Le temps des prisons. Inertie, réforme et reproduction d'un dispositif institutionnel » in Artières P., Lascoumes P (dir.)., Gouverner, enfermer. La prison, un modèle indépassable ?, Paris, Presses de Sciences Po, 2004, pp. 57-82.

74. Salle G., «Des “infimes matérialités carcérales” à “l’État de droit”. Prison et Rechtstaat en RFA depuis 1968 », Droit et Société, n69-70, 2008, pp. 507-518.

75. Chantraine G., "La prison post-disciplinaire », Déviance et Société, vol. 30, n³, 2006, pp. 273-288.

76. Chauvenet A., Orlic F. et Rostaing C., La violence carcérale en question, Paris, Presses universitaires de France, 2008.

77. Cardon C., «L'organisation de la sexualité en prison comme instrument de gestion des longues peines ", Revue internationale de criminologie et de police technique et scientifique, vol. 52, 1999, pp. 315-321 ; Lancelevée C., «Une sexualité à l'étroit. Les Unités de Visite Familiale et la réorganisation carcérale de l'intime ", Sociétés Contemporaines, vol. 3, n 83 , 2011, pp. 107-130.

78. Comfort M., “In The Tube At San Quentin. The 'Secondary Prisonization' of Women Visiting Inmates”, Journal of Contemporary Ethnography, vol. 32, n`1, 2003, pp. 77-107 ; Touraut C., La famille à l'épreuve de la prison, Paris, Presses Universitaires de France, 2012 ; Ricordeau G., Les détenus et leurs proche : solidarités et sentiments à l'ombre des murs, Paris, Éditions Autrement, 2008.

79. Combessie P., «Ouverture des prisons : jusqu’à quel point ? » in Veil C. et Lhuilier D. (eds.), La prison en changement, Ramonville Saint-Agne, Érès, 2000, pp. 69-99.

80. Le colloque international et pluridisciplinaire sur les «Pratiques, acteurs et espaces de l'enfermement : Circulations et transferts » s'est tenu à Berlin du $1^{\mathrm{er}}$ au 3 décembre 2011. Il était organisé par Falk Bretschneider, Mathilde Darley, Camille Lancelevée, Bénédicte Michalon et Thomas Scheffer en partenariat avec l'Université Humboldt (Berlin), le Centre Marc Bloch (Berlin), le programme de recherche TerrFerme (ANR / Conseil Région d'Aquitaine), le CRIA (EHESS), l'IRIS (EHESS) et l'Université franco-allemande. 
l'enfermement et ses murs. La confrontation d'analyses ancrées dans différents contextes historiques, politiques et sociaux révèle en effet la permanence de la dialectique ouverture / fermeture dans les politiques d'enfermement, et permet surtout d'en appréhender les effets paradoxaux sur les pratiques des acteurs, dans et hors les murs. Se dessine alors une cartographie complexe de la gestion des illégalismes par l'enfermement. 